\title{
Strongyloides stercoralis hyperinfestation syndrome with Escherichia coli meningitis: report of two cases
}

\author{
LESLEY A SMALLMAN, ${ }^{*}$ JENNIFER A YOUNG, ${ }^{*}$ WR SHORTLAND-WEBB, $\dagger$ \\ MP CAREY,* J MICHAEL $\ddagger$ \\ From the *Department of Pathology, The Medical School, University of Birmingham, the †Department of \\ Pathology, Dudley Road Hospital, Birmingham, and the $\ddagger$ Queen Elizabeth Hospital, Birmingham
}

SUMMARY Two cases of Strongyloides stercoralis hyperinfestation syndrome accompanied by Gram negative bacteraemia and meningitis were studied. Both occurred in non-immunosuppressed West Indian women.

Secondary Gram negative bacteraemia and meningitis has previously been documented in association with overwhelming strongyloidiasis. ${ }^{1}$ Most instances, however, have occurred in patients immunosuppressed by disease, treatment, or both. We report two cases of Escherichia coli meningitis and hyperinfestation with Strongyloides stercoralis in nonimmunosuppressed West Indian women.

\section{Case 1}

A 38 year old West Indian woman was admitted to Dudley Road Hospital, Birmingham, in December 1978. She had a two month history of anorexia, weight loss, abdominal pain, diarrhoea, and swollen legs. The patient had lived in England since 1960 but had returned home to Jamaica for a month in 1976. On examination she had pitting oedema of both legs up to the knees and a distended abdomen. Investigations showed a haemoglobin of $12.2 \mathrm{~g} / \mathrm{dl}$, a low serum total protein and albumin, and mildly raised liver function tests. A barium meal x-ray showed that the jejunum and ileum were grossly dilated; and there was slow progression of the contrast medium through the bowel but no localised abnormality.

In January 1979 her condition deteriorated rapidly and she became confused, delirious, dehydrated, and shocked. Her haemoglobin had fallen to $8.5 \mathrm{~g} / \mathrm{dl}$, and, although without fever, she had leucocytosis of $24 \times$ $10^{9} / 1$ without evidence of eosinophilia. Laparotomy was performed, but only thickening of the terminal ileum and enlarged mesenteric lymph nodes were found. Biopsy of the distal ileum, however, showed infestation with $S$ stercoralis (Fig. 1) and partial vil-

Accepted for publication 11 December 1985 lous atrophy; only reactive changes were seen in the mesenteric lymph nodes.

Postoperative recovery was poor, and ventilation was necessary after two respiratory arrests. Parasites were isolated from the stools and nasogastric, pharyngeal, and tracheal aspirates, and treatment with high dose thiobendazole was therefore started. Several days later she became feverish, and $E$ coli was grown from blood cultures. Treatment with gentamicin was started, but there was no improvement in her condition and she died later that month.

\section{NECROPSY FINDINGS AND HISTOLOGY}

At necropsy pitting oedema of both legs up to the knees and a recent abdominal scar were found. A few subcutaneous nodules, which, on incision, were found to be abscesses, were present on the left thigh. The lungs, although firm and oedematous, showed no evidence of pneumonia. Small bilateral pleural effusions were present and the peritoneal cavity contained one and a half litres of ascitic fluid. The small intestines appeared unremarkable apart from some serosal congestion in the distal portion where the biopsy had been obtained, and fibrinous adhesions were present between the adjacent loops of bowel. The liver $(2850 \mathrm{~g})$ was fatty, and small subcapsular abscesses were identified in the right kidney. The brain $(1060 \mathrm{~g})$ was soft with adherent dura. Pus had collected in the sulci on the lateral aspect of the right temporal lobe, and a basal meninigitis was found. A swab from one of the subcutaneous abscesses grew $E$ coli and Pseudomonas aeruginosa; the pus from the meninges grew only $E$ coli.

Microscopic examination showed that there was granulation tissue and a fibrinopurulent exudate on the serosal aspect of the small intestine, but no residual parasites could be identified. Ventilation 


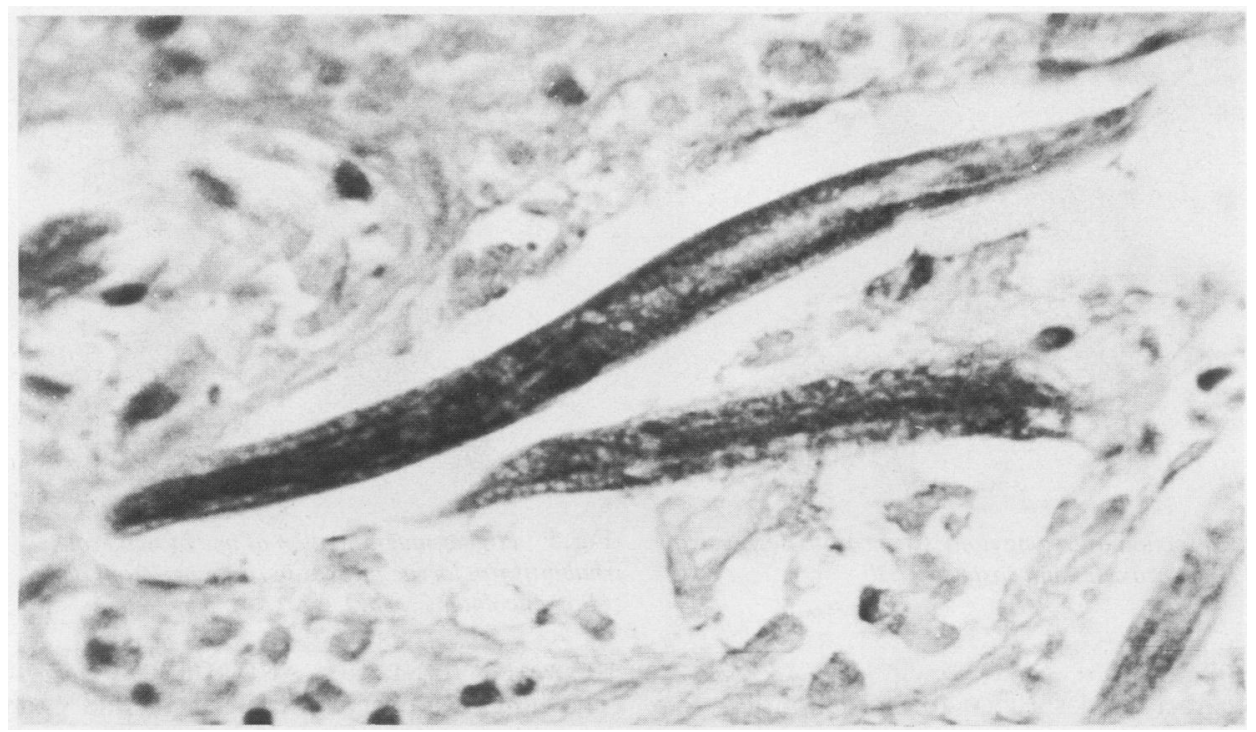

Fig. 1 Larvae of Strongyloides stercoralis embedded in wall of bowel. (Haematoxylin and eosin.) $\times 450$.

changes and a few granulomas were present in the lung but again no convincing parasites could be found. Sections of the brain showed meningitis and ventriculitis. The abscesses within the subcutaneous tissues and right kidney were confirmed.

\section{Case 2}

A 68 year old West Indian woman was admitted to the Queen Elizabeth Hospital, Birmingham, in September 1984 with a history of anorexia, weight loss, abdominal pain, drowsiness, and restlessness. On examination she was dehydrated and had abdominal tenderness. Investigations showed poor renal function, a metabolic acidosis and a haemoglobin of $7.6 \mathrm{~g} / \mathrm{dl}$ (hypochromic, normocytic). The cause of the anaemia was not discovered; there was no evidence of sickle haemoglobin or faecal occult blood, and a barium enema x-ray showed normal results. Renal function and the metabolic acidosis improved with intravenous fluids and bicarbonate, and she was discharged from hospital having been prescribed iron

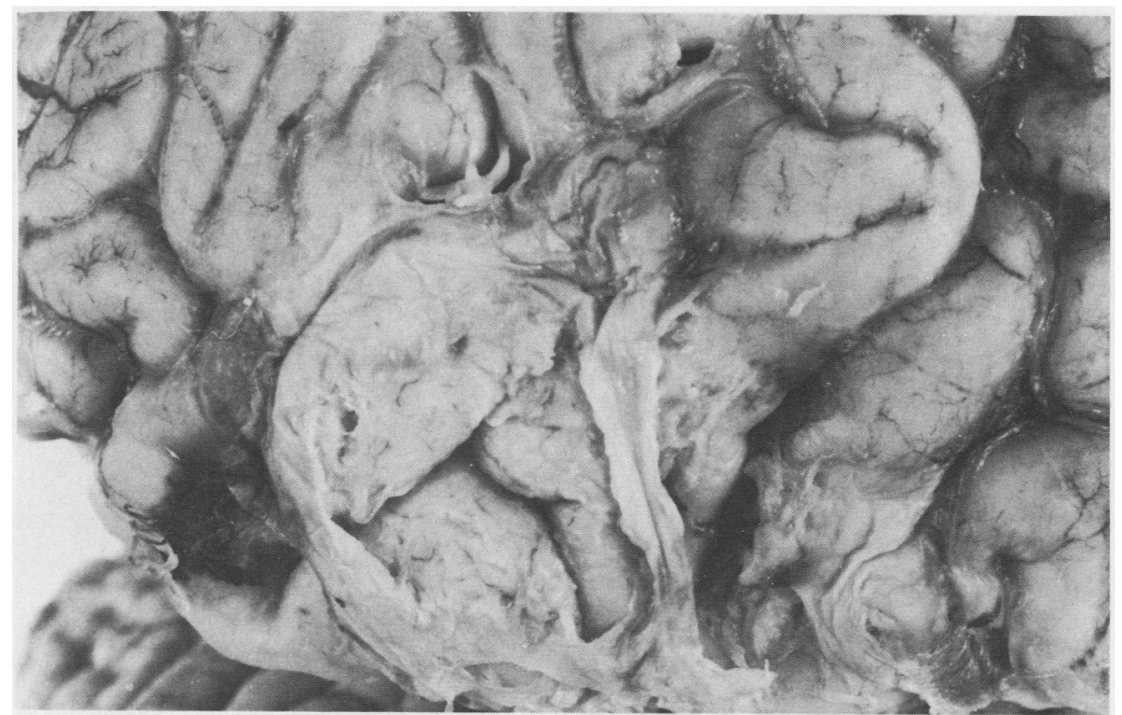

Fig. 2 Purulent exudate within the meninges over temporoparietal lobes of brain. 


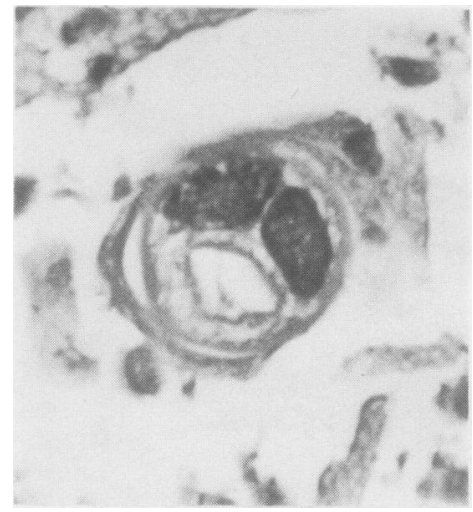

Fig. 3 Cross section of Strongyloides stercoralis seen in wall of bowel. (Haematoxylin and eosin.) $\times 620$.

supplements.

In December 1984 she had to be readmitted. She was now delirious, unresponsive, and feverish with a tachycardia. There was stiffness of the neck, and Kernig's sign was positive. Laboratory findings included peripheral blood leucocytosis of $9.3 \times 10^{9} / 1$ (no eosinophilia), a raised white cell count (neutrophils, polymorphs), a low glucose concentration, and occasional Gram negative bacilli in the cerebrospinal fluid. A heavy growth of $E$ coli was cultured. Despite treatment with chloramphenicol and cefataxime her condition deteriorated and she died three days after admission.

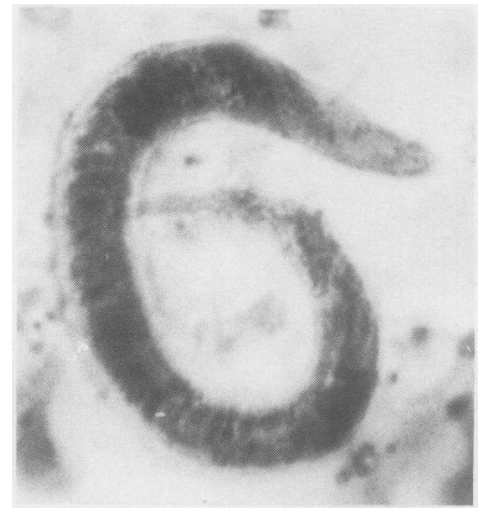

Fig. 5 Higher magnification of one of numerous rhabditiform larvae present in scrapings from bowel. (Papanicolaou.) $\times 480$.

\section{NECROPSY FINDINGS AND HISTOLOGY}

The lungs were heavy, congested, and oedematous but without evidence of pneumonia. There were small bilateral effusions. The stomach, and small and large intestines appeared macroscopically unremarkable. The liver $(1265 \mathrm{~g})$ was fatty, and there was mild acute pancreatitis. On dissecting the thyroid and parathyroids, abscesses were found within the strap muscles of the neck. The brain $(1245 \mathrm{~g})$ showed atrophic changes and pus in the subarachnoid space, particularly overlying the superior aspects of the left precentral and postcentral gyri (Fig. 2). The pus in the meninges contained moderate numbers of Gram

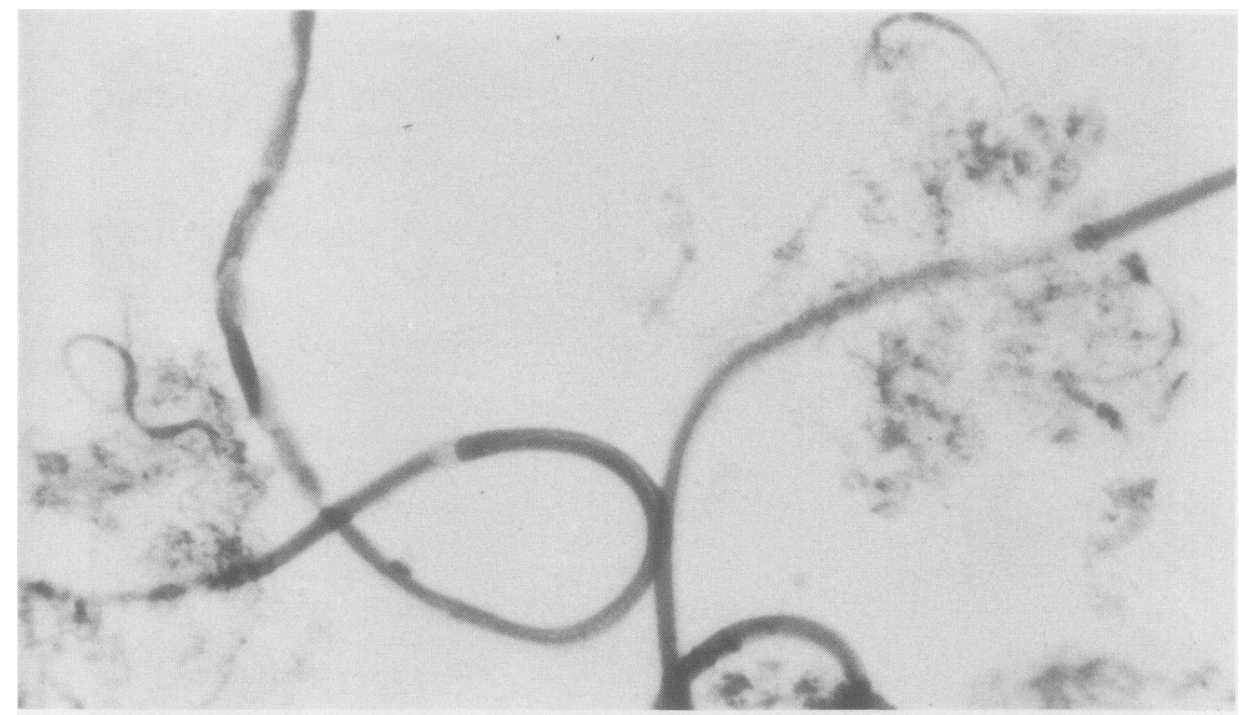

Fig. 4 Scraping from bowel wall containing two adult worms and many small larvae. (Papanicolaou.) $\times 120$. 
negative bacilli, and a heavy growth of $E$ coli was cultured.

Histological examination showed that the duodenum, jejunum, and ileum were honeycombed with adult worms, larvae, and eggs of $S$ stercoralis (Fig. 3). Larvae were present in the crypts of Lieberkuhn, the bowel wall, and the veins of the submucosa and serosa. Material was scraped off the mucosal surface of the fixed bowel, mixed with saline, and centrifuged. Smears were made from the deposit and stained by the Papanicolaou method. Complete adult worms (Fig. 4) and intact larvae (Fig. 5) were clearly identifiable. Sections of the brain showed a meningitis and ventriculitis, and Gram negative bacilli were shown within the purulent exudate. The abscesses within the strap muscles of the neck were confirmed. No parasites were seen in tissues outside the bowel.

\section{Discussion}

$S$ stercoralis was first isolated from the faeces of French colonial troops suffering from diarrhoea in Cochin-China (Vietnam) in $1876 .^{2}$ It is an intestinal nematode that infests a large proportion of the world's population but is particularly common in warm moist climates. ${ }^{3}$

Filariform larvae within the soil penetrate the skin, enter lymphatics and veins, and are thus carried to the lungs. After migrating up the trachea to the glottis they are swallowed and reach the duodenum and jejunum. Here the larvae burrow into the gut mucosa and become hermaphrodite adult females, able to lay up to 40 eggs a day. The ova develop into rhabditiform larvae, which enter the bowel, and are expelled in the faeces. As they traverse the intestinal tract some metamorphose into infective filariform larvae. These invade the gut wall or perianal skin gaining access to the bloodstream, thus establishing an endogenous cycle. This recycling process, known as "autoinfestation" is unique to $S$ stercoralis and may account for the persistence of the parasite even 40 years after the host has left the area where the infestation was acquired. ${ }^{34}$ Unfortunately, no information concerning residence was available for case 2 , but case 1 had lived in England since 1960 returning to Jamaica for one month only in 1976. The parasite was presumably acquired in the West Indies in both cases and had persisted due to autoinfestation.

The normal host parasite equilibrium with the nematode seems to be predominantly maintained by cell mediated immunity. ${ }^{56}$ Breakdown of these defences due to disease or immunosuppression may result in hyperinfestation (augmentation of the normal life cycle), or disseminated infestation (spread to organs not normally part of the life cycle). ${ }^{1}$ Overwhelming infestation can, however, develop in nor- mal hosts. ${ }^{1}$ The mechanisms of pathogenesis are not well understood; general debility and protein-calorie malnutrition have been implicated, ${ }^{5}$ but malabsorption, protein losing enteropathy, and hypoproteinaemia may be produced by $S$ stercoralis itself. ${ }^{7}$ Both our patients had hyperinfestation with $S$ stercoralis. In case 2 parasites were found only in sections of the small intestine at necropsy. In case 1, however, larvae were seen in a surgical biopsy and were also isolated from stools and nasogastric, pharyngeal, and tracheal aspirates. This patient had two respiratory arrests, and larvae were also most probably present within the lungs. Treatment with thiobendazole seemed to be successful as no parasites were seen in the sections of bowel or lungs at necropsy, although there were granulomata in the lungs. No cause of immunosuppression was found in either case.

Hyperinfestation and disseminated infestation may be accompanied by persistent or recurrent bacteraemia due to organisms of enteric origin. ${ }^{1}$ It has been suggested that these bacteria gain access to the circulation by one or all of three mechanisms. Firstly, disruption of the bowel wall could provide entrance to the circulation for faecal flora. ${ }^{8-11}$ Secondly, it is also thought more likely that bacteria adhere to the external surface of larvae and are transported from the gut in piggy back fashion. ${ }^{12}$ Finally, bacteria may be excreted into the circulation from the intestinal tract of migrating larvae. ${ }^{12}$

Only a few cases of $S$ stercoralis hyperinfestation syndrome had been documented before 1964. In 1981 Igra-Siegman et al described two cases, one of which was accompanied by Klebsiella pneumoniae bacteraemia and meningitis. The same authors reviewed 103 other cases published since 1964. Eighty nine of the patients were immunosuppressed by disease, treatment, or both; the 14 others had no apparent immune deficiency. Thirty two of the immunosuppressed patients were included in a report that was based on necropsy material; sepsis due to Gram negative bacteria was mentioned in three of these cases, but, unfortunately, no further details were given. ${ }^{5}$ Thirty of the residual 71 cases reviewed had a secondary bacterial infection, 25 of whom died-11 with meningitis.

Spread to the brain is extremely rare, even in disseminated strongyloides, but published examples include one case each of meningitis, ${ }^{13}$ brain abscess, ${ }^{14}$ and cerebral infarction. ${ }^{15}$

Both of our patients had a Gram negative ( $E$ coli) meningitis. In addition, there were subcutaneous and renal abscesses in case 1 , and abscesses in the strap muscles of the neck in case 2 . The first patient's condition deteriorated shortly before laparotomy. Although $E$ coli was only isolated from the blood culture several days after the operation, and disruption 
of the bowel wall during biopsy may have resulted in the Gram negative bacteraemia, the onset of the symptoms and signs does not suggest this. It seems more likely that the infection occurred either as a result of bacteria adhering to the external surface of larvae or to bacteria being excreted into the circulation from the intestinal tract of migrating larvae. One or both of these mechanisms are also the likely explanation for the Gram negative bacteraemia in case 2. No larvae were found in the meninges or brain parenchyma in either case.

Secondary Gram negative bacteraemia and meningitis developing in association with strongyloidiasis is considered to be rare and occurs almost exclusively in immunocompromised subjects. Our two cases show that the hyperinfestation syndrome should be considered even in non-immunosuppressed immigrant patients with Gram negative bacteraemia, meningitis, and unexplained anaemia.

We thank Dr A Paton for permission to report case 1. Our thanks are also due to Miss $M$ Trumper for technical help, $\mathrm{Mr}$ A Cooper for help in preparing the photomicrographs, and Miss A Wright for typing the manuscript.

\section{References}

${ }^{1}$ Igra-Siegman Y, Kapila R, Sen P, Kaminski ZC, Louria BD. Syndrome of hyperinfection with Strongyloides stercoralis. Rev Infect Dis 1981;3:397-407.
${ }^{2}$ Normand A. Sur la maladie dite diarrhée de Cochin-chine. $C R$ Séaonces Acad Sci (III) 1876;83:316-8.

${ }^{3}$ Grove DI. Strongyloidiasis. In: Warren KS, Mohamoud AAF, eds. Tropical and geographical medicine. New York: McGraw-Hill 1984:373-9.

${ }^{4}$ Grove DI. Strongyloidiasis in allied ex-prisones of war in southeast Asia. Br Med J 1980;280:598-601.

${ }^{5}$ Purtilo DR, Meyers WM, Connor DH. Fatal strongyloidiasis in immunosuppressed patients. Am J Med 1974;56:488-93.

${ }^{6}$ Dwork KG, Jaffe JR, Lieberman HD. Strongyloidiasis with massive hyperinfection. NY State J Med 1975;75:1230-4.

${ }^{7}$ Liepman M. Strongyloides stercoralis, a complication of immunosuppression. JAMA 1975;231:387-8.

${ }^{8}$ De Paola D, Dias LB, Da Silva JR. Enteritis due to Strongyloides stercoralis. A report of five fatal cases. Am J Dig Dis 1962;7:1086-98.

${ }^{9}$ Faust EC, DeGroat A. Internal autoinfection in human strongyloidiasis. Am J Trop Med 1940;20:359-71.

${ }^{10}$ Meyers AM, Shapiro DJ, Milne FJ, Myburgh JA, Robkin R. Strongloides stercoralis hyperinfection in a renal allograft recipient. S Afr Med J 1976;50:1301-2.

${ }^{11}$ Rivera E, Maldonado N, Veléz-García E, Grillo AJ. Malaret G. Hyperinfection syndrome with Strongyloides stercoralis. Ann Intern Med 1970;72:199-204.

${ }^{12}$ Brown HW, Perna VP. An overwhelming strongyloides infection. JAMA 1958;168:648-51.

${ }^{13}$ Owar R, Wamukota WM. A fatal case of strongyloidiasis with strongyloides larvae in the meninges. Trans $R$ Soc Trop Med Hyg 1970;70:497-9.

${ }^{14}$ Neefe LI, Pinilla I, Garagusi VF, Bauer H. Disseminated strongyloidiasis with cerebral involvement. A complication of corticosteroid therapy. Am J Med 1973;55:832-8.

${ }^{15}$ Masdeu JC, Trantulavanich S, Gorelick PP, et al. Brain abscess caused by Stronglyoides stercoralis. Arch Neurol 1982;39:62-3.

Requests for reprints to: Dr Lesley A Smallman, Department of Pathology, The Medical School, University of Birmingham, Birmingham B15 2TJ, England. 